\title{
LIGHT MESONS AND INFRARED BEHAVIOR OF THE RUNNING COUPLING CONSTANT IN QCD
}

\author{
M. BALDICCHI AND G. M. PROSPERI \\ Dipartimento di Fisica dell'Università di Milano; I.N.F.N. Sez. di Milano; \\ via Celoria 16, I20133 Milano, Italy; E-mail: prosperi@mi.infn.it
}

\begin{abstract}
A previous method for handling bound states in QCD is briefly revisited. Taking advantage of the Feynman-Schwinger representation for the iterated quark propagator in an external field, it is possible to give closed representations for certain appropriate (second order) two point and four point Green functions, $H^{(2)}(x-y)$ and $H^{(4)}\left(x_{1}, x_{2}, y_{1}, y_{2}\right)$, as path integrals on quark world lines. Then, starting from reasonable assumptions on the Wilson line correlators, a Bethe-Salpeter equation for $H^{(4)}$ and a Dyson-Schwinger equation for $H^{(2)}$ can be obtained, which are consistent with the Goldstone theorem in the chiral limit. Such equations are too complicate to be solved directly. However, a reduced Salpeter equation can be derived which is tractable and has been applied to a calculation of the meson spectrum. The results are in general good agreement with the data, but with the important exceptions of the light pseudo scalars (that are related to the breaking of the chiral symmetry). In this scenario two important improvements can be introduced: a) the fixed coupling constant can be replaced by a running coupling constant $\alpha_{\mathrm{S}}\left(Q^{2}\right)$ appropriately modified in the infrared region; b) the fixed mass in the reduced equation can be replaced for light quarks by an effective mass depending on the momentum of the particle, as suggested by the form of the DS equation. Then even the light pseudo scalar mesons can be made to agree with to their experimental value.
\end{abstract}

\section{Introduction}

In previous papers we have introduced what we have called a second order Bethe-Salpeter formalism, which works in terms of certain appropriate four point and two point Green functions, $H^{(4)}\left(x_{1}, x_{2}, y_{1}, y_{2}\right)$ and $H^{(2)}(x-y)^{1}$. Taking advantage of a Feynman-Schwinger representation for the "second order" quark propagator in an external field, it is possible to write $H^{(2)}$ and $H^{(4)}$ as path integrals on quark or antiquark world lines joining $y$ to $x, y_{1,2}$ to $x_{1,2}$. In such representations the gauge field appears only trough Wilson line correlators like $\frac{1}{\sqrt{3}} \operatorname{Tr}_{\text {color }}\left\langle\exp \left[i \int_{y}^{x} d z^{\mu} A_{\mu}(z)\right]\right.$ or $\frac{1}{3} \operatorname{Tr}_{\text {color }}\left\langle\exp \left[i \int_{y_{1}}^{x_{1}} d z^{\mu} A_{\mu}(z)\right] \exp \left[i \int_{x_{2}}^{y_{2}} d z^{\nu} A_{\nu}(z)\right]\right\rangle$ which reduce to ordinary Wilson loops $W$ in the limit $x \rightarrow y$ or $x_{1} \rightarrow x_{2}$ and $y_{1} \rightarrow y_{2}$. In analogy 
with a usual assumption on $W$ such correlators are written as the sum of their perturbative expressions and appropriate area terms and it is possible to obtain a confining Bethe-Salpeter equation for the quantity $H^{(4)}$ and a corresponding Dyson-Schwinger equation for $H^{(2)}$.

The above equations are too complicate to be solved directly. However, by a conventional three dimensional reduction, one can derive a tractable squared mass operator that can be applied to an evaluation of the spectrum $^{2}$. With an appropriate choice of the parameters (quark masses, coupling constant, string tension) the results can be made in good agreement with the data in the entire framework of the light-light, light-heavy and heavy-heavy quark-antiquark sectors, with the important exception, however, of the light pseudo scalar mesons ${ }^{3}$.

In this paper we want to discuss two important improvements to the above scenario:

a) the fixed coupling constant $\alpha_{\mathrm{s}}$ is replaced by a running coupling constant $\alpha_{\mathrm{s}}\left(Q^{2}\right)$, which amounts to take into account higher order contributions in $\alpha_{\mathrm{s}}$ (and in particular closed quark loops);

b) an effective mass for the light quarks is introduced which depends on the momentum of the particle, as suggested by the form of the DS equation.

As well known, the usual perturbative expressions for $\alpha_{\mathrm{s}}\left(Q^{2}\right)$ have an unphysical singularity for $Q^{2}=\Lambda_{\mathrm{QCD}}^{2}$, which would be disastrous for our purpose. However, various modification have been proposed for the infrared region ${ }^{4,5}$. We have considered in particular the Shirkov-Solovtsov prescription, which rests only on general analyticity requirements. We find that with such prescription, and parametrazing the effective squared light quark masses by a simple polynomial, even the light pseudo scalar mesons can be made in agreement with their experimental values. It should be stressed that in the fit only the quark masses are treated as free parameters, while the constant $\Lambda$ and $\sigma$ occurring in the BS kernel are a priori fixed on the basis of high energy phenomenology and lattice simulations. Results in this line were already published in ref. $\left.{ }^{[6}\right]$; here we present a more systematic study.

The remaining part of the paper is organized as the following plan: in sections 2 and 3 we briefly revue the second order Bethe-Salpeter formalism, in section 4 we discuss the running coupling constant and effective mass, in sections 5 and 6 we reports the results and make some conclusions. 


\section{Second order correlators}

After integrating out the fermionic fields, the appropriate ordinary (first order) four point function for the quark-antiquark bound state problem can be written

$$
\begin{aligned}
G^{(4)}\left(x_{1}, x_{2} ; y_{1}, y_{2}\right) & =\frac{1}{3} \sum_{a b}\left\langle 0\left|\mathrm{~T} \psi_{1 a}\left(x_{1}\right) \bar{\psi}_{2 a}\left(x_{2}\right) \bar{\psi}_{1 b}\left(y_{1}\right) \psi_{2 b}\left(y_{2}\right)\right| 0\right\rangle= \\
& =-\frac{1}{3} \operatorname{Tr}_{\text {Color }}\left\langle S_{1}\left(x_{1}, y_{1} ; A\right) S_{2}\left(y_{2}, x_{2} ; A\right)\right\rangle,
\end{aligned}
$$

Here $a$ and $b$ are color indexes, the subscripts 1 and 2 refer to the quark and the antiquark respectively, projection on the color singlet has been performed, $S(x, y ; A)$ denotes the quark propagator in an external field,

$$
\left(i \gamma^{\mu} D_{\mu}-m\right) S(x, y ; A)=\delta^{4}(x-y),
$$

and

$$
\langle f[A]\rangle=\int D A M_{F}[A] e^{i S_{G}[A]} f[A],
$$

with

$$
M_{F}[A]=\operatorname{Det}_{j=1}^{2}\left[1+g \gamma^{\mu} A_{\mu}\left(i \gamma_{j}^{\nu} \partial_{j \nu}-m_{j}\right)^{-1}\right] .
$$

The second order four point function is defined by

$$
H^{(4)}\left(x_{1}, x_{2} ; y_{1}, y_{2}\right)=-\frac{1}{3} \operatorname{Tr}_{\mathrm{color}}\left\langle\Delta_{1}^{\sigma}\left(x_{1}, y_{1} ; A\right) \Delta_{2}^{\sigma}\left(y_{2}, x_{2} ; A\right)\right\rangle,
$$

where $\Delta_{1}^{\sigma}(x, y ; A)$ is the second order propagator that satisfies the second order differential equation

$$
\left(D_{\mu} D^{\mu}+m^{2}-\frac{1}{2} g \sigma^{\mu \nu} F_{\mu \nu}\right) \Delta^{\sigma}(x, y ; A)=-\delta^{4}(x-y),
$$

$\left(\sigma^{\mu \nu}=\frac{i}{2}\left[\gamma^{\mu}, \gamma^{\nu}\right]\right)$ and is related to $S(x, y ; A)$ by

$$
S(x, y ; A)=\left(i \gamma^{\nu} D_{\nu}+m\right) \Delta^{\sigma}(x, y ; A) .
$$

The quantity $H^{(4)}$ is related to $G^{(4)}$ by an integro-differential operator, of the type $\left[\left(i \gamma_{1}^{\mu} \partial_{1 \mu}+m_{1}\right)\left(i \gamma_{2}^{\nu} \partial_{2 \nu}+m_{2}\right)+\ldots\right]$, which we do not need to specify in detail. The important fact is that the two functions are completely equivalent for the determination of the bound states since their Fourier transforms $\hat{H}^{(4)}$ and $\hat{G}^{(4)}$ have related analyticity properties and the same poles. 
The advantage in considering second order quantities is that it is possible to write for $\Delta^{\sigma}(x, y ; A)$ a generalized Feynman-Schwinger representation, i. e. to solve eq. (5) in terms of a quark path integral

$$
\begin{gathered}
\Delta^{\sigma}(x, y ; A)=-\frac{i}{2} \int_{0}^{\infty} d s \int_{y}^{x} D z \exp \left[-i \int_{0}^{s} d \tau \frac{1}{2}\left(m^{2}+\dot{z}^{2}\right)\right] \\
S_{0}^{s} \mathrm{P} \exp \left[i g \int_{0}^{s} d \tau \dot{z}^{\mu} A_{\mu}(z)\right]
\end{gathered}
$$

where the world-line $z^{\mu}=z^{\mu}(\tau)$ connecting $y$ to $x$ is written in the fourdimensional language in terms of an additional parameter $\tau, S_{0}^{s}=\mathrm{T} \exp [-$ $\left.\frac{1}{4} \int_{0}^{s} d \tau \sigma^{\mu \nu} \frac{\delta}{\delta S^{\mu \nu}(z)}\right]$ and $\delta S^{\mu \nu}=d z^{\mu} \delta z^{\nu}-d z^{\nu} \delta z^{\mu}$ (the functional derivative being defined through an arbitrary deformation, $z \rightarrow z+\delta z$, of the worldline).

Replacing eq. (7) in eq. (4) a similar representation can be obtained for the 4-point function

$$
\begin{gathered}
H^{(4)}\left(x_{1}, x_{2} ; y_{1}, y_{2}\right)=\left(\frac{1}{2}\right)^{2} \int_{0}^{\infty} d s_{1} \int_{0}^{\infty} d s_{2} \int_{y_{1}}^{x_{1}} D z_{1} \int_{y_{2}}^{x_{2}} D z_{2} \\
\exp \left\{-\frac{i}{2} \int_{0}^{s_{1}} d \tau_{1}\left(m_{1}^{2}+\dot{z}_{1}^{2}\right)-\frac{i}{2} \int_{0}^{s_{2}} d \tau_{2}\left(m_{2}^{2}+\dot{z}_{2}^{2}\right)\right\} \\
\frac{1}{3} S_{0}^{s_{1}} S_{0}^{s_{2}} \operatorname{Tr}_{\text {color }}\left\langle\mathrm{P} \exp \left\{i g \int_{y_{1}}^{x_{1}} d z_{1}^{\mu} A_{\mu}\left(z_{1}\right)\right\} \mathrm{P} \exp \left\{i g \int_{x_{2}}^{y_{2}} d z_{2}^{\mu} A_{\mu}\left(z_{2}\right)\right\}\right\rangle,
\end{gathered}
$$

The interesting aspect of the above equation is that the gauge field appears in it only through the expectation value of the product of the two Wilson lines.

Similarly the second order two point function (uncolored full quark propagator) $H^{(2)}(x-y)=\frac{i}{\sqrt{3}} \operatorname{Tr}_{\text {color }}\langle S(x, y: A)\rangle$ can be written

$$
\begin{aligned}
H^{(2)}(x-y)= & \frac{1}{2} \int_{0}^{\infty} d s \int_{y}^{x} D z \exp \left\{-\frac{i}{2} \int_{0}^{s} d \tau\left(m^{2}+\dot{z}^{2}\right)\right\} \\
& \frac{1}{\sqrt{3}} S_{0}^{s} \operatorname{Tr}_{\text {color }}\left\langle\mathrm{P} \exp \left\{i g \int_{y}^{x} d z^{\mu} A_{\mu}(z)\right\}\right\rangle
\end{aligned}
$$

Notice that, for large separations, we can also write in operatorial form

$$
H^{(4)}\left(x_{1}, x_{2} ; y_{1}, y_{2}\right)=\frac{1}{3} \sum_{a b}\left\langle 0\left|\mathrm{~T} \phi_{1 a}\left(x_{1}\right) \bar{\psi}_{2 a}\left(x_{2}\right) \bar{\psi}_{1 b}\left(y_{1}\right) \phi_{2 b}\left(y_{2}\right)\right| 0\right\rangle
$$

and

$$
H^{(2)}(x-y)=\sum_{a}\left\langle 0\left|\mathrm{~T} \phi_{a}(x) \bar{\psi}_{a}(y)\right| 0\right\rangle
$$


$\phi(x)$ being defined by

$$
\psi(x)=\left(i \gamma^{\nu} D_{\nu}+m\right) \phi(x) .
$$

The above forms are important for a definition of the relativistic wave function.

\section{Bethe-Salpeter and Dyson-Schwinger equations}

In the limit $x_{2} \rightarrow x_{1}, y_{2} \rightarrow y_{1}$ or $y \rightarrow x$ the two Wilson lines occurring in (8) or the single line occurring in (9) close in a single Wilson loop $\Gamma$

$$
W=\left\langle\frac{1}{3} \operatorname{Tr}_{\text {color }} \mathrm{P} \exp \left[\oint_{\Gamma} d z^{\mu} A_{\mu}(z)\right]\right\rangle .
$$

As in the previous papers we assume that in a first approximation $i \ln W$ can be written as the sum of its perturbative expression and an area term $i \ln W=(i \ln W)_{\text {pert }}+\sigma S$. Then, at the lowest order in the coupling constant, we can assume

$$
\begin{aligned}
& i \ln W=\frac{4}{3} g^{2} \oint d z^{\mu} \oint d z^{\nu \prime} D_{\mu \nu}\left(z-z^{\prime}\right)+ \\
& \sigma \oint d z^{0} \oint d z^{0 \prime} \delta\left(z^{0}-z^{0 \prime}\right)\left|\mathbf{z}-\mathbf{z}^{\prime}\right| \int_{0}^{1} d \lambda\left\{1-\left[\lambda \frac{d \mathbf{z}_{\mathrm{T}}}{d z^{0}}+(1-\lambda) \frac{d \mathbf{z}_{\mathrm{T}}^{\prime}}{d z^{0 \prime}}\right]^{2}\right\}^{\frac{1}{2}} .
\end{aligned}
$$

Notice that the surface term in (11) is written as the algebraic sum of successive equal time straight strips $\left(\mathbf{z}_{\mathrm{T}}\right.$ denotes the transversal component of $\mathbf{z}$ ). For a flat loop or for other special geometries (e. g. for two quarks uniformly rotating around their fixed center of mass) this coincides obviously with the plane or the minimum surface delimited by $\Gamma$. That is not generally the case, indeed the right hand side of (11) usually depends on the reference frame. Since, however, in contrast e. g. with $S_{\min }$, such quantity maintains many of the analytic properties of the original $i \ln W$, we shall assume (11) to be valid for an arbitrary loop in the center of mass

reference frame. Actually we shall assume this even for $x_{2} \neq x_{1}, y_{2} \neq y_{1}$ or $y \neq x$, in analogy with what happens in the pure perturbative case. In this way single perturbative and confinement contributions are put on the same foot and we may refer to them as a gluon exchange and a string connection between the two quarks.

Replacing (11) in (8) and (9) we obtain the following equations

$$
H^{(4)}\left(x_{1}, x_{2} ; y_{1}, y_{2}\right)=\left(\frac{1}{2}\right)^{2} \int_{0}^{\infty} d s_{1} \int_{0}^{\infty} d s_{2} \int_{y_{1}}^{x_{1}} D z_{1} \int_{y_{2}}^{x_{2}} D z_{2}
$$




$$
\begin{aligned}
& \exp \left\{-\frac{i}{2} \sum_{j=1}^{2} \int_{0}^{s_{j}} d \tau_{j}\left(m_{j}^{2}+\dot{z}_{j}^{2}\right)\right\} S_{0}^{s_{1}} S_{0}^{s_{2}} \exp \left\{i \sum_{j=1}^{2} \int_{0}^{s_{j}} d \tau_{j}\right. \\
& \left.\int_{0}^{\tau_{j}} d \tau_{j}^{\prime} E\left(z_{j}-z_{j}^{\prime} ; \dot{z}_{j}, \dot{z}_{j}^{\prime}\right)-i \int_{0}^{s_{1}} d \tau_{1} \int_{0}^{s_{2}} d \tau_{2} E\left(z_{1}-z_{2} ; \dot{z}_{1}, \dot{z}_{2}\right)\right\}
\end{aligned}
$$

and

$$
\begin{array}{r}
H^{(2)}(x-y)=\frac{1}{2} \int_{0}^{\infty} d s \int_{y}^{x} D z \exp \left\{-\frac{i}{2} \int_{0}^{s} d \tau\left(m^{2}+\dot{z}^{2}\right)\right\} \\
S_{0}^{s} \exp \left\{i \int_{0}^{s} \int_{0}^{\tau} E\left(z-z^{\prime} ; \dot{z}, \dot{z}^{\prime}\right)\right\}
\end{array}
$$

where we have set

$$
E\left(\zeta ; p, p^{\prime}\right)=E_{\text {pert }}\left(\zeta ; p, p^{\prime}\right)+E_{\text {conf }}\left(\zeta ; p, p^{\prime}\right)
$$

with

$$
\left\{\begin{array}{l}
E_{\mathrm{pert}}=4 \pi \frac{4}{3} \alpha_{\mathrm{s}} D_{\mu \nu}(\zeta) p^{\mu} p^{\prime \nu} \\
E_{\mathrm{conf}}=\delta\left(\zeta_{0}\right)|\zeta| \epsilon\left(p_{0}\right) \epsilon\left(p_{0}^{\prime}\right) \int_{0}^{1} d \lambda\left\{p_{0}^{2} p_{0}^{\prime 2}-\left[\lambda p_{0}^{\prime} \mathbf{p}_{\mathrm{T}}+(1-\lambda) p_{0} \mathbf{p}_{\mathrm{T}}^{\prime}\right]^{2}\right\}^{\frac{1}{2}}
\end{array}\right.
$$

From eqs. (12) and (13), by various manipulation and using an appropriate iterative procedure, a Bethe-Salpeter equation for the function $H^{(4)}\left(x_{1}, x_{2} ; y_{1}, y_{2}\right)$ and a Dyson-Schwinger equation for $H^{(2)}(x-y)$ can be derived in a kind of generalized ladder and rainbow approximation respectively in the form

$$
\begin{aligned}
H^{(4)}\left(x_{1}, x_{2} ; y_{1}, y_{2}\right) & =H_{1}^{(2)}\left(x_{1}-y_{1}\right) H_{2}^{(2)}\left(x_{2}-y_{2}\right)- \\
& -i \int d^{4} \xi_{1} d^{4} \xi_{2} d^{4} \eta_{1} d^{4} \eta_{2} H_{1}^{(2)}\left(x_{1}-\xi_{1}\right) H_{2}^{(2)}\left(x_{2}-\xi_{2}\right) \\
& \quad \times I_{a b}\left(\xi_{1}, \xi_{2} ; \eta_{1}, \eta_{2}\right) \sigma_{1}^{a} \sigma_{2}^{b} H^{(4)}\left(\eta_{1}, \eta_{2} ; y_{1}, y_{2}\right)
\end{aligned}
$$

and

$$
\begin{aligned}
H^{(2)}(x-y)=H_{0}^{(2)} & (x-y)+i \int d^{4} \xi d^{4} \eta d^{4} \xi^{\prime} d^{4} \eta^{\prime} H_{0}^{(2)}(x-\xi) \\
& \times I_{a b}\left(\xi, \xi^{\prime} ; \eta, \eta^{\prime}\right) \sigma^{a} H^{(2)}\left(\eta-\eta^{\prime}\right) \sigma^{b} H^{(2)}\left(\xi^{\prime}-y\right),
\end{aligned}
$$

where $a, b=0, \mu \nu$, we have set $\sigma^{0}=1$, and $H_{1}^{(2)}$ and $H_{2}^{(2)}$ denote the second order full quark and the antiquark propagators respectively.

In momentum representation, the corresponding homogeneous BSequation can be written

$$
\begin{array}{r}
\Phi_{P}(k)=-i \int \frac{d^{4} u}{(2 \pi)^{4}} \hat{I}_{a b}\left(k-u, \frac{1}{2} P+\frac{k+u}{2}, \frac{1}{2} P-\frac{k+u}{2}\right) \\
\hat{H}_{1}^{(2)}\left(\frac{1}{2} P+k\right) \sigma^{a} \Phi_{P}(u) \sigma^{b} \hat{H}_{2}^{(2)}\left(-\frac{1}{2} P+k\right),
\end{array}
$$


the center of mass frame has to be understood, $P=\left(m_{B}, \mathbf{0}\right)$ and $\Phi_{P}(k)$ denotes the appropriate second order wave function

$$
\left\langle 0\left|\phi\left(\frac{\xi}{2}\right) \bar{\psi}\left(-\frac{\xi}{2}\right)\right| P\right\rangle=\frac{1}{(2 \pi)^{2}} \Phi_{P}(k) e^{-i k \xi} .
$$

Similarly, in terms of the irreducible self-energy, defined by

$$
\hat{H}^{(2)}(k)=\frac{i}{k^{2}-m^{2}}+\frac{i}{k^{2}-m^{2}} i \Gamma(k) \hat{H}^{(2)}(k),
$$

the DS-equation can be written also

$$
\hat{\Gamma}(k)=\int \frac{d^{4} l}{(2 \pi)^{4}} \hat{I}_{a b}\left(k-l ; \frac{k+l}{2}, \frac{k+l}{2}\right) \sigma^{a} \hat{H}^{(2)}(l) \sigma^{b} .
$$

The kernels in (18) and (19) are the same in the two equations, consistently with the requirement of chiral symmetry limit ${ }^{7}$, and are given by

$$
\begin{aligned}
& \hat{I}_{0 ; 0}\left(Q ; p, p^{\prime}\right)=4 \int d^{4} \zeta e^{i Q \zeta} E\left(\zeta ; p, p^{\prime}\right)=16 \pi \frac{4}{3} \alpha_{\mathrm{s}} p^{\alpha} p^{\prime \beta} \hat{D}_{\alpha \beta}(Q)+ \\
& +4 \sigma \int d^{3} \zeta e^{-i \mathbf{Q} \cdot \zeta}|\zeta| \epsilon\left(p_{0}\right) \epsilon\left(p_{0}^{\prime}\right) \int_{0}^{1} d \lambda\left\{p_{0}^{2} p_{0}^{\prime 2}-\left[\lambda p_{0}^{\prime} \mathbf{p}_{\mathrm{T}}+(1-\lambda) p_{0} \mathbf{p}_{\mathrm{T}}^{\prime}\right]^{2}\right\}^{\frac{1}{2}} \\
& \hat{I}_{\mu \nu ; 0}\left(Q ; p, p^{\prime}\right)=4 \pi i \frac{4}{3} \alpha_{\mathrm{s}}\left(\delta_{\mu}^{\alpha} Q_{\nu}-\delta_{\nu}^{\alpha} Q_{\mu}\right) p_{\beta}^{\prime} \hat{D}_{\alpha \beta}(Q)- \\
& -\sigma \int d^{3} \zeta e^{-i \mathbf{Q} \cdot \zeta} \epsilon\left(p_{0}\right) \frac{\zeta_{\mu} p_{\nu}-\zeta_{\nu} p_{\mu}}{|\zeta| \sqrt{p_{0}^{2}-\mathbf{p}_{\mathrm{T}}^{2}}} p_{0}^{\prime} \\
& \hat{I}_{0 ; \rho \sigma}\left(Q ; p, p^{\prime}\right)=-4 \pi i \frac{4}{3} \alpha_{\mathrm{s}} p^{\alpha}\left(\delta_{\rho}^{\beta} Q_{\sigma}-\delta_{\sigma}^{\beta} Q_{\rho}\right) \hat{D}_{\alpha \beta}(Q)+ \\
& +\sigma \int d^{3} \zeta e^{-i \mathbf{Q} \cdot \zeta} p_{0} \frac{\zeta_{\rho} p_{\sigma}^{\prime}-\zeta_{\sigma} p_{\rho}^{\prime}}{|\zeta| \sqrt{p_{0}^{\prime 2}-\mathbf{p}_{\mathrm{T}}^{\prime 2}}} \epsilon\left(p_{0}^{\prime}\right) \\
& \hat{I}_{\mu \nu ; \rho \sigma}\left(Q ; p, p^{\prime}\right)=\pi \frac{4}{3} \alpha_{\mathrm{s}}\left(\delta_{\mu}^{\alpha} Q_{\nu}-\delta_{\nu}^{\alpha} Q_{\mu}\right)\left(\delta_{\rho}^{\alpha} Q_{\sigma}-\delta_{\sigma}^{\alpha} Q_{\rho}\right) \hat{D}_{\alpha \beta}(Q),
\end{aligned}
$$

where in the second and in the third equation $\zeta_{0}=0$ has to be understood.

To find the $q \bar{q}$ spectrum, in principle one should solve first (19) and use the resulting propagator in (18). In practice this turns out to be a difficult task and one has to resort to the three dimensional equation which can be obtained from (18) by the so called instantaneous approximation. This consists in replacing $\hat{H}_{j}^{(2)}(k)$ in (18) with the free quark propagator $\frac{i}{k^{2}-m_{j}^{2}}$ and the kernels $\hat{I}_{a b}$ with its so called instantaneous approximation $\hat{I}_{a b}^{\mathrm{inst}}\left(\mathbf{k}, \mathbf{k}^{\prime}\right)^{1}$. 
The reduced equation takes the form of the eigenvalue equation for a squared mass operator $M^{2}=M_{0}^{2}+U$, with $M_{0}=\sqrt{m_{1}^{2}+\mathbf{k}^{2}}+\sqrt{m_{2}^{2}+\mathbf{k}^{2}}$ and

$$
\left\langle\mathbf{k}|U| \mathbf{k}^{\prime}\right\rangle=\frac{1}{(2 \pi)^{3}} \sqrt{\frac{w_{1}+w_{2}}{2 w_{1} w_{2}}} \hat{I}_{a b}^{\text {inst }}\left(\mathbf{k}, \mathbf{k}^{\prime}\right) \sqrt{\frac{w_{1}^{\prime}+w_{2}^{\prime}}{2 w_{1}^{\prime} w_{2}^{\prime}}} \sigma_{1}^{a} \sigma_{2}^{b}
$$

(for an explicit expression see ref. $\left[{ }^{6,3}\right]$ ). The quadratic form of the above equation obviously derives from the second order formalism we have used.

Alternatively, in more usual terms, one can look for the eigenvalue of the mass operator or center of mass Hamiltonian $H_{\mathrm{CM}} \equiv M=M_{0}+V$, with $V$ defined by $M_{0} V+V M_{0}+V^{2}=U$. Neglecting the term $V^{2}$, the linear potential $V$ can be obtained from $U$ simply by the kinematic replacement $\sqrt{\frac{\left(w_{1}+w_{2}\right)\left(w_{1}^{\prime}+w_{2}^{\prime}\right)}{w_{1} w_{2} w_{1}^{\prime} w_{2}^{\prime}}} \rightarrow \frac{1}{2 \sqrt{w_{1} w_{2} w_{1}^{\prime} w_{2}^{\prime}}}$. Such expression is particularly useful for a comparison with models based on potential. In particular in the static limit $V$ reduces to the Cornell potential

$$
V=-\frac{4}{3} \frac{\alpha_{\mathrm{s}}}{r}+\sigma r
$$

in the semirelativistic limit (up to $\frac{1}{m^{2}}$ terms after an appropriate FoldyWouthuysen transformation) equals the potential discussed in ref. [ $\left.{ }^{8}\right]$. If the spin dependent terms are neglected but full relativistic kinematics is kept, it becomes identical to the potential of the (relativistic) flux tube model $^{1}$.

\section{Running coupling constant and effective mass}

In ref. $\left.{ }^{3}\right]$ the spectrum was evaluated for both the operators $M^{2}$ and $H_{\mathrm{CM}}$ introduced in the preceding section, omitting the spin-orbit terms in the potential but including the hyperfine ones. As we told, with fixed coupling constant and quark masses, a general good fit of the data was obtained over the entire calculable spectrum with, however, the relevant exception of the light pseudoscalar mesons. At the light of the idea that such mesons should be Goldston massless particles in the chiral limit, this is not surprising ${ }^{7}$. The results obtained in ref. [6] suggest, however, that the situation can be greatly improved using a running coupling constant and an effective light quark mass function of the momentum, as implied in (19) for a kernel of the type (20).

At one loop the running coupling constant in QCD is usually written as

$$
\alpha_{\mathrm{s}}\left(Q^{2}\right)=\frac{4 \pi}{\beta_{0} \ln \left(Q^{2} / \Lambda^{2}\right)}
$$


$Q$ being the relevant energy scale, $\beta_{0}=11-\frac{2}{3} N_{\mathrm{f}}$, and $N_{\mathrm{f}}$ the number of flavors with masses smaller than $Q$. Such expression becomes singular and completely inadequate as $Q^{2}$ approaches $\Lambda^{2}$.

The most naive modification of eq. (23) consists in saturating $\alpha_{\mathrm{s}}\left(Q^{2}\right)$ to a certain maximum value $\bar{\alpha}_{\mathrm{s}}$ as $Q^{2}$ decrease (fig. 1) and in treating this value as a phenomenological parameter (truncation prescription).

Alternatively Shirkov and Solovtsov ${ }^{5}$ replace (23) with

$$
\alpha_{\mathrm{s}}\left(Q^{2}\right)=\frac{4 \pi}{\beta_{0}}\left(\frac{1}{\ln \left(Q^{2} / \Lambda^{2}\right)}+\frac{\Lambda^{2}}{\Lambda^{2}-Q^{2}}\right) .
$$

This remains regular for $Q^{2}=\Lambda^{2}$ and has a finite $\Lambda$ independent limit, $\alpha_{\mathrm{s}}(0)=4 \pi / \beta_{0}$, for $Q^{2} \rightarrow 0$. Eq. (24) is obtained assuming a dispersion relation with a cut from $-\infty<Q^{2}<0$ and applying (23) to the evaluation of the spectral function alone.

In the quark-antiquark bound state problem the variable $Q^{2}$ can be identified with the squared momentum transfer $\mathbf{Q}^{2}=\left(\mathbf{k}-\mathbf{k}^{\prime}\right)^{2}$. Then, typically, $\left\langle\mathbf{Q}^{2}\right\rangle$ ranges between $(0.1 \mathrm{GeV})^{2}$ and $(1 \mathrm{GeV})^{2}$ for different quark masses and internal excitations and values of $\mathbf{Q}^{2}$ smaller than $\Lambda^{2}$ can be important. The specific infrared behavior is therefore expected to affect the spectrum and other properties of mesons.

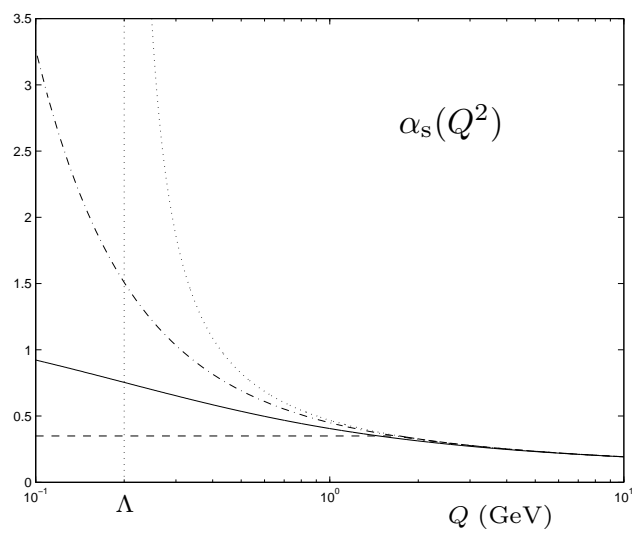

Figure 1. Running coupling constant $\alpha_{\mathrm{S}}\left(Q^{2}\right)$ on logarithmic scale. Perturbative expression (dotted line), truncation prescription (dashed line), Shirkov-Solovtsov prescription (full line), Nesterenko prescription (dot-dashed line).

Coming to the quark masses, notice that, even neglecting the spin dependent part in $\hat{H}^{(2)}(k)$ (consistently with what we do for $\left\langle\mathbf{k}|U| \mathbf{k}^{\prime}\right\rangle$ ), we 
could more sensibly write $\hat{H}^{(2)}(k)=i /\left(k^{2}-m^{2}-\Gamma_{0}(k)\right)$ in the reduction procedure, rather than simply identify such quantity with its free expression $\left(\Gamma_{0}(k)=0\right)$. However, since the $I_{a}\left(Q, p, p^{\prime}\right)$ are not formally covariant, but given in terms of C.M. variables, $\Gamma_{0}(k)$ must depend separately on $k_{0}^{2}$ and $\mathbf{k}^{2}$. Then the pole of $H_{2}(k)$, defined by

$$
k_{0}^{2}-\mathbf{k}^{2}-m^{2}-\Gamma\left(k_{0}^{2}, \mathbf{k}^{2}\right)=0,
$$

could be written

$$
k_{0}^{2}=m_{\text {eff }}^{2}(|\mathbf{k}|)+\mathbf{k}^{2},
$$

where $m_{\text {eff }}^{2}(|\mathbf{k}|)$ would be a $|\mathbf{k}|$ dependent expression that is expected to approach the current $m^{2}$ for $|\mathbf{k}| \rightarrow 0$ and to increase toward a kind of constituent $m^{\prime 2}$ as $|\mathbf{k}|$ increases $^{\text {a }}$. Then, eventually, we obtain the same operator $M^{2}$ as given above, but with $m_{1}$ and $m_{2}$ replaced by expressions like $m_{\text {eff }}^{2}(|\mathbf{k}|)$.

As matter of fact we have not tried in this paper to evaluate $\Gamma(p)$ from first principles by solving eq. (19) but have simply parametrized $m_{\text {eff }}^{2}(|\mathbf{k}|)$ as a polynomial in $|\mathbf{k}|$ for the light quarks and used a fixed mass as in the preceding papers for strange and heavy quarks.

\section{Numerical procedure and results}

In the calculation of the eigenvalues of the operator $M^{2}$ introduced in sect. 4 we have used the same approximations and the same numerical procedure as in the previous papers. We have retained only the hyperfine part of the complicate spin dependent terms (spin orbit and tensorial ones) occurring in the potential $U$. We have solved the eigenvalue equation for $H_{\mathrm{CM}}$ with $V$ equal its static limit (22) by the Rayleigh-Ritz method, using the three dimensional Harmonic oscillator basis and diagonalizing a $30 \times 30$ matrix. Then we have evaluated $\left\langle\phi_{n}\left|M^{2}\right| \phi_{n}\right\rangle$ for the eigenfunction $\phi_{n}$ obtained in the first step.

In figs. 2 and 3 we have represented graphically the results already obtained in ref. $\left[{ }^{3}\right]$ with a truncated running coupling constant (crosses) and the new ones performed with the Shirkov-Solovtsov prescription (circlets), contrasted with the data (small lines) $\left.{ }^{9}\right]$. In tables I-VII we give the corresponding numerical values.

a Take into account that from the virial theorem for a linear potential in the extreme relativistic approximation we have $\langle|\mathbf{k}|\rangle=\sigma\langle r\rangle$ and a large $|\mathbf{k}|$ corresponds to a peripheral interaction or, what is the same, to a small $|\mathbf{Q}|$. 
$(\mathrm{GeV})$
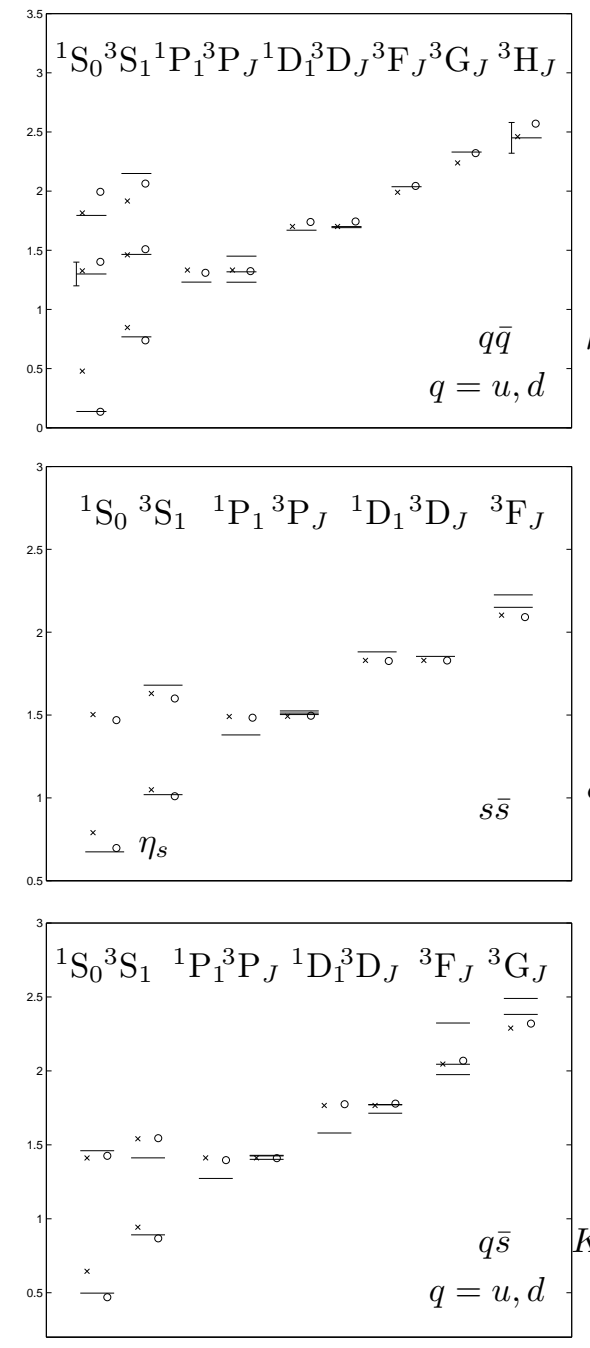
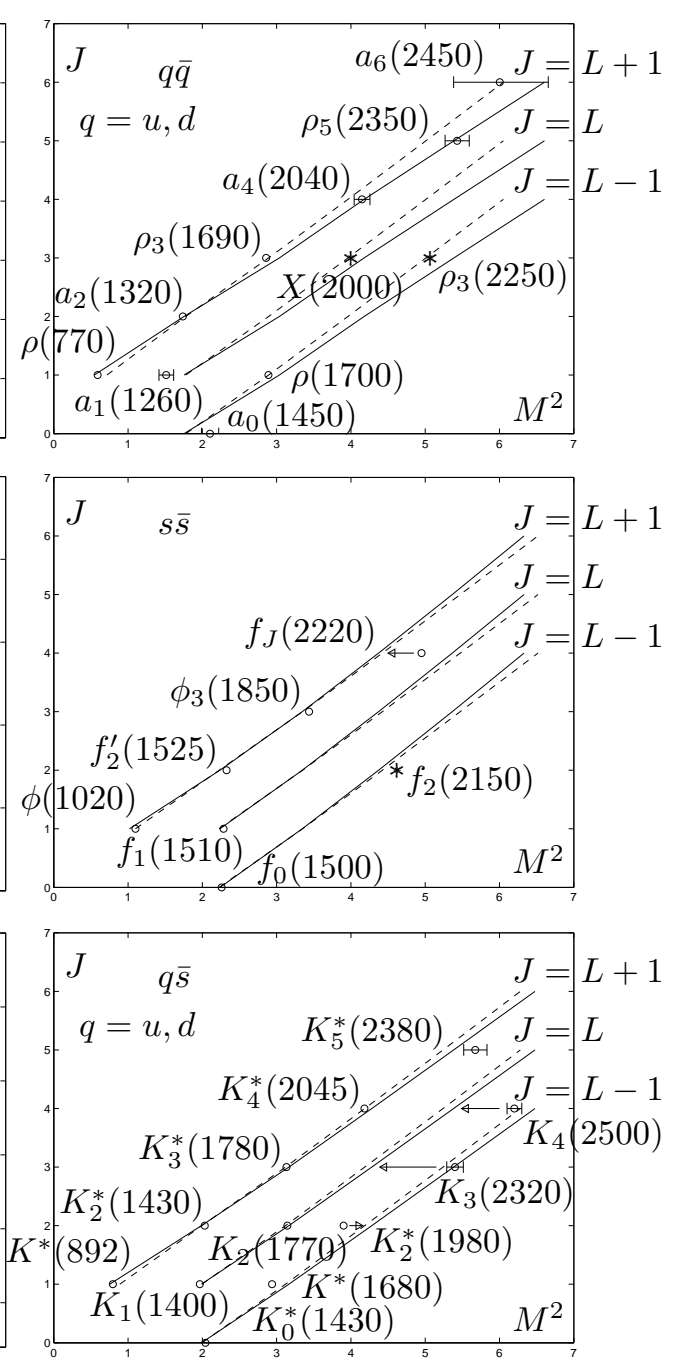

Figure 2. Light-light quarkonium spectrum and Regge trajectories. $n_{f}=4, \Lambda=$ $0.2 \mathrm{GeV}$. Crosses and dashed line: truncation prescription, $\bar{\alpha}_{\mathrm{s}}=0.35, \sigma=0.2 \mathrm{GeV}^{2}$, $m_{s}=0.2 \mathrm{GeV}, m_{u}=m_{d}=0.01 \mathrm{GeV}$. Circlets and full line: Shirkov-Solovtsov $\alpha_{\mathrm{s}}\left(Q^{2}\right)$, $\sigma=0.18 \mathrm{GeV}^{2}, m_{s}=0.381 \mathrm{GeV}, m_{u}^{2}=m_{d}^{2}=0.121 k-0.025 k^{2}+0.25 k^{4}$.

In both cases we have taken $N_{\mathrm{f}}=4$ and $\Lambda=200 \mathrm{MeV}$. With the truncation prescription, the light quark masses were fixed on typical current values, $m_{u}=m_{d}=10 \mathrm{MeV}, m_{s}=200 \mathrm{MeV}$, the maximum value for $\alpha_{\mathrm{s}}\left(Q^{2}\right)$, the string tension and the heavy quark masses were treated as 


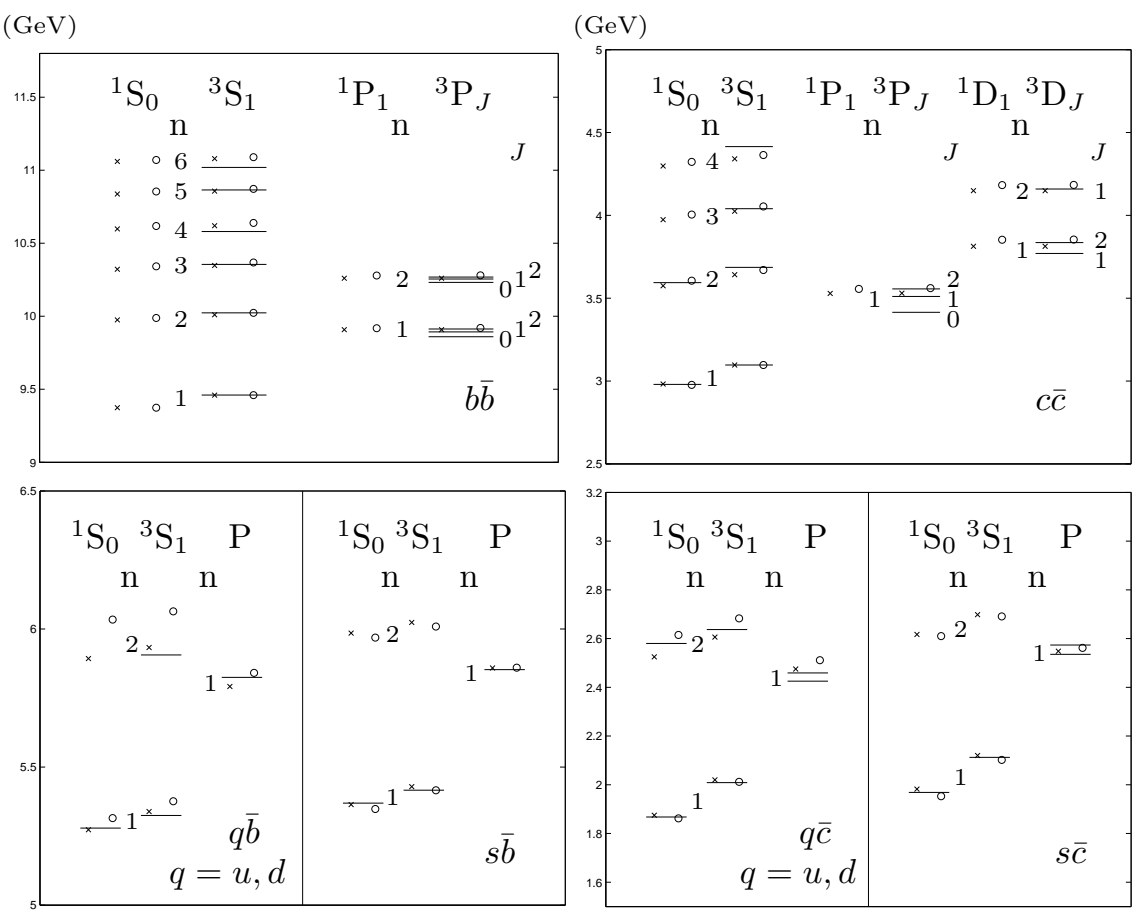

Figure 3. Heavy-heavy and heavy-light quarkonium spectrum. $n_{f}=4, \Lambda=0.2 \mathrm{GeV}$. Crosses: truncation prescription, $\bar{\alpha}_{\mathrm{s}}=0.35, \sigma=0.2 \mathrm{GeV}^{2}, m_{b}=4.763 \mathrm{GeV}, m_{c}=$ $1.394 \mathrm{GeV}, m_{s}=0.2 \mathrm{GeV}, m_{u}=m_{d}=0.01 \mathrm{GeV}$. Circlets: Shirkov-Solovtsov $\alpha_{\mathrm{s}}\left(Q^{2}\right)$, $\sigma=0.18 \mathrm{GeV}^{2}, m_{b}=4.898, m_{c}=1.545, m_{s}=0.381 \mathrm{GeV}, m_{u}^{2}=m_{d}^{2}=0.121 k-$ $0.025 k^{2}+0.25 k^{4}$.

adjustable parameters and chosen as $\bar{\alpha}_{\mathrm{s}}=0.35, \sigma=0.2 \mathrm{GeV}^{2}$ (in order to reproduce the correct $J / \Psi-\eta_{c}$ separation and Regge trajectory slope) and $m_{c}=1.394 \mathrm{GeV}, m_{b}=4.763 \mathrm{GeV}$ (in order to obtain exact masses for $J / \Psi$ and $\Upsilon$ ). On the contrary, with the Shirkov-Solovtsov prescription, we have fixed the string tension on the commonly accepted value $\sigma=0.18 \mathrm{GeV}^{2}$ and used all masses as adjustable parameters. For the light quarks we have taken (in $\mathrm{GeV}^{2}$ )

$$
m_{u}^{2}=m_{d}^{2}=0.121 k-0.025 k^{2}+0.25 k^{4},
$$

( $k$ being the quark momentum in the meson C. M. frame), for the strange and the heavy quarks $m_{s}=0.381 \mathrm{GeV}, m_{c}=1.545 \mathrm{GeV}$ and $m_{b}=4.898 \mathrm{GeV}$.

As it is apparent the use of a running coupling constant with infrared truncation does not improve essentially the situation in comparison with 
Table I. $q \bar{q}(q=u, d), n_{f}=4, \Lambda=0.2 \mathrm{GeV}$. (a) truncation prescription $\bar{\alpha}_{\mathrm{s}}=0.35, \sigma=0.2 \mathrm{GeV}^{2}, m_{u}=m_{d}=0.01 \mathrm{GeV}$.

(b) Shirkov-Solovtsov $\alpha_{\mathrm{s}}\left(Q^{2}\right), \sigma=0.18 \mathrm{GeV}^{2}$, $m_{u}^{2}=m_{d}^{2}=0.121 k-0.025 k^{2}+0.25 k^{4}$.

\begin{tabular}{|c|c|c|c|c|}
\hline States & & $\begin{array}{c}\text { experimental values } \\
(\mathrm{MeV})\end{array}$ & $\begin{array}{c}(\mathrm{a}) \\
(\mathrm{MeV})\end{array}$ & $\begin{array}{c}(\mathrm{b}) \\
(\mathrm{MeV})\end{array}$ \\
\hline $1{ }^{1} \mathrm{~S}_{0}$ & $\left\{\begin{array}{l}\pi^{0} \\
\pi^{ \pm}\end{array}\right.$ & $\begin{array}{c}134.9764 \pm 0.0006 \\
139.56995 \pm 0.00035\end{array}$ & 479 & 135 \\
\hline $1^{3} \mathrm{~S}_{1}$ & $\rho(770)$ & $768.5 \pm 0.6$ & 846 & 739 \\
\hline $1 \Delta \mathrm{SS}$ & & 630 & 367 & 604 \\
\hline $2{ }^{1} \mathrm{~S}_{0}$ & $\pi(1300)$ & $1300 \pm 100$ & 1326 & 1402 \\
\hline $2{ }^{3} \mathrm{~S}_{1}$ & $\rho(1450)$ & $1465 \pm 25$ & 1461 & 1509 \\
\hline $2 \Delta \mathrm{SS}$ & & 165 & 135 & 107 \\
\hline $3{ }^{1} \mathrm{~S}_{0}$ & $\pi(1800)$ & $1795 \pm 10$ & 1815 & 1994 \\
\hline $3{ }^{3} \mathrm{~S}_{1}$ & $\rho(2150)$ & $2149 \pm 17$ & 1916 & 2064 \\
\hline $3 \Delta \mathrm{SS}$ & & 354 & 101 & 70 \\
\hline $1^{1} \mathrm{P}_{1}$ & $b_{1}(1235)$ & $1231 \pm 10$ & 1333 & 1310 \\
\hline $1^{3} \mathrm{P}_{2}$ & $a_{2}(1320)$ & $1318.1 \pm 0.7)$ & & \\
\hline $1^{3} \mathrm{P}_{1}$ & $a_{1}(1260)$ & $1230 \pm 40 \quad 1303$ & 1333 & 1324 \\
\hline $1^{3} \mathrm{P}_{0}$ & $a_{0}(1450)$ & $1450 \pm 40$ & & \\
\hline $1^{1} \mathrm{D}_{2}$ & $\pi_{2}(1670)$ & $1670 \pm 20$ & 1701 & 1739 \\
\hline $1^{3} \mathrm{D}_{3}$ & $\rho_{3}(1690)$ & $1691 \pm 5$ & & \\
\hline $\begin{array}{l}1^{3} \mathrm{D}_{2} \\
1^{3} \mathrm{D}_{1}\end{array}$ & $\rho(1700)$ & $1700 \pm 20$ & 1701 & 1743 \\
\hline $\begin{array}{l}1{ }^{1} \mathrm{~F}_{3} \\
1^{3} \mathrm{~F}_{4}\end{array}$ & $a_{4}(2040)$ & $2037 \pm 26$ & 1990 & 2043 \\
\hline $\begin{array}{l}1^{3} \mathrm{~F}_{3} \\
1^{3} \mathrm{~F}_{2}\end{array}$ & $X(2000)$ & & 1990 & 2044 \\
\hline $\begin{array}{l}1^{1} \mathrm{G}_{4} \\
1^{3} \mathrm{G}_{5}\end{array}$ & $\rho_{5}(2350)$ & $2330 \pm 35$ & 2238 & 2318 \\
\hline $\begin{array}{l}1{ }^{3} \mathrm{G}_{4} \\
1{ }^{3} \mathrm{G}_{3}\end{array}$ & $\rho_{3}(2250)$ & & 2238 & 2321 \\
\hline $\begin{array}{l}1{ }^{1} \mathrm{H}_{5} \\
1^{3} \mathrm{H}_{6} \\
1^{3} \mathrm{H}_{5} \\
1^{3} \mathrm{H}_{4}\end{array}$ & $a_{6}(2450)$ & $2450 \pm 130)$ & 2460 & 2570 \\
\hline
\end{tabular}


Table II. $s \bar{s}, n_{f}=4, \Lambda=0.2 \mathrm{GeV}$. (a) truncation prescription $\bar{\alpha}_{\mathrm{s}}=0.35$, $\sigma=0.2 \mathrm{GeV}^{2}, m_{s}=0.2 \mathrm{GeV}$. (b) Shirkov-Solovtsov $\alpha_{\mathrm{s}}\left(Q^{2}\right)$, $\sigma=0.18 \mathrm{GeV}^{2}, m_{s}=0.381 \mathrm{GeV}$.

\begin{tabular}{|c|c|c|c|c|}
\hline States & & $\begin{array}{c}\text { experimental values } \\
(\mathrm{MeV})\end{array}$ & $\begin{array}{c}(\mathrm{a}) \\
(\mathrm{MeV})\end{array}$ & $\begin{array}{c}(\mathrm{b}) \\
(\mathrm{MeV})\end{array}$ \\
\hline${ }^{1} \mathrm{~S}_{0}$ & $\eta_{\mathrm{s}}$ & $\left\{\begin{array}{c}\eta(547) \\
\eta^{\prime}(958)\end{array}\right\} 674$ & 790 & 697 \\
\hline $1^{3} \mathrm{~S}_{1}$ & $\phi(1020)$ & $1019.413 \pm 0.008$ & 1049 & 1010 \\
\hline $1 \Delta \mathrm{SS}$ & & $335.3 \pm 0.1$ & 259 & 313 \\
\hline $2{ }^{1} \mathrm{~S}_{0}$ & & & 1503 & 1469 \\
\hline $2{ }^{3} \mathrm{~S}_{1}$ & $\phi(1680)$ & $1680 \pm 20$ & 1630 & 1600 \\
\hline $2 \Delta \mathrm{SS}$ & & & 127 & 131 \\
\hline $3{ }^{1} \mathrm{~S}_{0}$ & & & 1977 & 1934 \\
\hline $3{ }^{3} \mathrm{~S}_{1}$ & & & 2068 & 2023 \\
\hline $3 \Delta \mathrm{SS}$ & & & 91 & 89 \\
\hline $1{ }^{1} \mathrm{P}_{1}$ & $h_{1}(1380)$ & $1380 \pm 20$ & 1491 & 1484 \\
\hline $1^{3} \mathrm{P}_{2}$ & $f_{2}^{\prime}(1525)$ & $1525 \pm 5$ & & \\
\hline $1^{3} \mathrm{P}_{1}$ & $f_{1}(1510)$ & $1512 \pm 4\} 1518$ & 1492 & 1496 \\
\hline $1^{3} \mathrm{P}_{0}$ & $f_{0}(1500)$ & $1503 \pm 11$ & & \\
\hline $1{ }^{1} \mathrm{D}_{2}$ & $\eta_{2}(1870)$ & $1881 \pm 32 \pm 40$ & 1830 & 1826 \\
\hline $1^{3} \mathrm{D}_{3}$ & $\phi_{3}(1850)$ & $1854 \pm 7)$ & & \\
\hline $\begin{array}{l}1^{3} \mathrm{D}_{2} \\
1^{3} \mathrm{D}_{1}\end{array}$ & & & 1830 & 1829 \\
\hline $1{ }^{1} \mathrm{~F}_{3}$ & & & 2103 & 2089 \\
\hline $\begin{array}{l}1^{3} \mathrm{~F}_{4} \\
1^{3} \mathrm{~F}_{3} \\
1^{3} \mathrm{~F}_{2}\end{array}$ & $\begin{array}{l}f_{J}(2220) \\
f_{2}(2150)\end{array}$ & $2225 \pm 6$ ) & 2103 & 2091 \\
\hline $\begin{array}{l}1{ }^{1} \mathrm{G}_{4} \\
1{ }^{3} \mathrm{G}_{5}\end{array}$ & & ) & 2339 & 2313 \\
\hline $\begin{array}{l}1{ }^{3} \mathrm{G}_{4} \\
1^{3} \mathrm{G}_{3}\end{array}$ & & & 2339 & 2316 \\
\hline $\begin{array}{l}1{ }^{1} \mathrm{H}_{5} \\
1^{3} \mathrm{H}_{6}\end{array}$ & & & 2553 & 2516 \\
\hline $\begin{array}{l}1^{3} \mathrm{H}_{5} \\
1^{3} \mathrm{H}_{4}\end{array}$ & & & 2553 & 2516 \\
\hline
\end{tabular}

the fixed coupling constant case. The same happen with other prescription like the Dokshitzer et al. prescription ${ }^{4}$. On the contrary with the 
Table III. $q \bar{s}(q=u, d), n_{f}=4, \Lambda=0.2 \mathrm{GeV}$. (a) truncation prescription $\bar{\alpha}_{\mathrm{s}}=0.35, \sigma=0.2 \mathrm{GeV}^{2}, m_{s}=0.2 \mathrm{GeV}, m_{u}=m_{d}=0.01 \mathrm{GeV}$.

(b) Shirkov-Solovtsov $\alpha_{\mathrm{s}}\left(Q^{2}\right), \sigma=0.18 \mathrm{GeV}^{2}, m_{s}=0.381 \mathrm{GeV}$, $m_{u}^{2}=m_{d}^{2}=0.121 k-0.025 k^{2}+0.25 k^{4}$.

\begin{tabular}{|c|c|c|c|c|}
\hline \multicolumn{2}{|l|}{ States } & $\begin{array}{c}\text { experimental values } \\
(\mathrm{MeV})\end{array}$ & \multirow{2}{*}{$\begin{array}{c}\begin{array}{c}(\mathrm{a}) \\
(\mathrm{MeV})\end{array} \\
645\end{array}$} & \multirow{2}{*}{$\begin{array}{c}\begin{array}{c}(\mathrm{b}) \\
(\mathrm{MeV})\end{array} \\
470\end{array}$} \\
\hline${ }^{1} \mathrm{~S}_{0}$ & $\left\{\begin{array}{l}K^{0} \\
K^{ \pm}\end{array}\right.$ & $\left.\begin{array}{l}497.672 \pm 0.031 \\
493.677 \pm 0.016\end{array}\right\}$ & & \\
\hline $1^{3} \mathrm{~S}_{1}$ & $K^{*}(892)$ & $891.59 \pm 0.24$ & 943 & 868 \\
\hline $1 \Delta \mathrm{SS}$ & & $393.9 \pm 0.3$ & 298 & 398 \\
\hline $2{ }^{1} \mathrm{~S}_{0}$ & $K(1460)$ & & 1411 & 1426 \\
\hline $2^{3} \mathrm{~S}_{1}$ & $K^{*}(1410)$ & $1412 \pm 12$ & 1541 & 1545 \\
\hline $2 \Delta \mathrm{SS}$ & & & 130 & 119 \\
\hline $3{ }^{1} \mathrm{~S}_{0}$ & & & 1892 & 1956 \\
\hline $3{ }^{3} \mathrm{~S}_{1}$ & & & 1989 & 2035 \\
\hline $3 \Delta \mathrm{SS}$ & & & 97 & 80 \\
\hline $1{ }^{1} \mathrm{P}_{1}$ & $K_{1}(1270)$ & $1273 \pm 7$ & 1412 & 1397 \\
\hline $1^{3} \mathrm{P}_{2}$ & $K_{2}^{*}(1430)$ & $1425.4 \pm 1.3$ ) & & \\
\hline $1^{3} \mathrm{P}_{1}$ & $K_{1}(1400)$ & $1402 \pm 7\} 1418$ & 1412 & 1410 \\
\hline $1^{3} \mathrm{P}_{0}$ & $K_{0}^{*}(1430)$ & $1429 \pm 5$ & & \\
\hline $1{ }^{1} \mathrm{D}_{2}$ & $K_{2}(1580)$ & & 1766 & 1775 \\
\hline $1^{3} \mathrm{D}_{3}$ & $K_{3}^{*}(1780)$ & $1770 \pm 10)$ & & \\
\hline $1{ }^{3} \mathrm{D}_{2}$ & $K_{2}(1770)$ & $1773 \pm 8\} 1765$ & 1766 & 1779 \\
\hline $1^{3} \mathrm{D}_{1}$ & $K^{*}(1680)$ & $1714 \pm 20$ & & \\
\hline $1{ }^{1} \mathrm{~F}_{3}$ & & & 2046 & 2067 \\
\hline $1^{3} \mathrm{~F}_{4}$ & $K_{4}^{*}(2045)$ & $2045 \pm 9)$ & & \\
\hline $1{ }^{3} \mathrm{~F}_{3}$ & $K_{3}(2320)$ & $2324 \pm 24\} 2130$ & 2046 & 2069 \\
\hline $1{ }^{3} \mathrm{~F}_{2}$ & $K_{2}^{*}(1980)$ & $1975 \pm 22$ & & \\
\hline $1{ }^{1} \mathrm{G}_{4}$ & & & 2289 & 2317 \\
\hline $1{ }^{3} \mathrm{G}_{5}$ & $K_{5}^{*}(2380)$ & $2382 \pm 14 \pm 19$ & & \\
\hline $1{ }^{3} \mathrm{G}_{4}$ & $K_{4}(2500)$ & $2490 \pm 20$ & 2289 & 2320 \\
\hline $1{ }^{3} \mathrm{G}_{3}$ & & & & \\
\hline $1{ }^{1} \mathrm{H}_{5}$ & & & 2506 & 2545 \\
\hline $1^{3} \mathrm{H}_{6}$ & & & & \\
\hline $1{ }^{3} \mathrm{H}_{5}$ & & & 2506 & 2545 \\
\hline $1^{3} \mathrm{H}_{4}$ & & & & \\
\hline
\end{tabular}


Table IV. $b \bar{b}, n_{f}=4, \Lambda=0.2 \mathrm{GeV}$. (a) truncation prescription $\bar{\alpha}_{\mathrm{s}}=0.35$, $\sigma=0.2 \mathrm{GeV}^{2}, m_{b}=4.763 \mathrm{GeV}$. (b) Shirkov-Solovtsov $\alpha_{\mathrm{s}}\left(Q^{2}\right)$, $\sigma=0.18 \mathrm{GeV}^{2}, m_{b}=4.898 \mathrm{GeV}$.

\begin{tabular}{|c|c|c|c|c|c|}
\hline States & & \multicolumn{2}{|c|}{$\begin{array}{c}\text { experimental values } \\
(\mathrm{MeV})\end{array}$} & $\begin{array}{c}\text { (a) } \\
(\mathrm{MeV})\end{array}$ & $\begin{array}{c}(\mathrm{b}) \\
(\mathrm{MeV}) \\
\end{array}$ \\
\hline $1{ }^{1} \mathrm{~S}_{0}$ & & \multirow{3}{*}{\multicolumn{2}{|c|}{$9460.30 \pm 0.26$}} & 9374 & 9374 \\
\hline $1{ }^{3} \mathrm{~S}_{1}$ & $\Upsilon(1 S)$ & & & 9460 & 9460 \\
\hline $2{ }^{1} \mathrm{~S}_{0}$ & & & & 9975 & 9988 \\
\hline $2{ }^{3} \mathrm{~S}_{1}$ & $\Upsilon(2 S)$ & \multirow{2}{*}{\multicolumn{2}{|c|}{$10023.26 \pm 0.31$}} & 10010 & 10023 \\
\hline $3{ }^{1} \mathrm{~S}_{0}$ & & & & 10322 & 10342 \\
\hline $3{ }^{3} \mathrm{~S}_{1}$ & $\Upsilon(3 S)$ & \multirow{2}{*}{\multicolumn{2}{|c|}{$10355.2 \pm 0.5$}} & 10348 & 10368 \\
\hline $4{ }^{1} \mathrm{~S}_{0}$ & & & & 10598 & 10618 \\
\hline $4{ }^{3} \mathrm{~S}_{1}$ & $\Upsilon(4 S)$ & \multirow{2}{*}{\multicolumn{2}{|c|}{$10580.0 \pm 3.5$}} & 10620 & 10639 \\
\hline $5{ }^{1} \mathrm{~S}_{0}$ & & & & 10837 & 10854 \\
\hline $5^{3} \mathrm{~S}_{1}$ & $\Upsilon(10860)$ & \multirow{2}{*}{\multicolumn{2}{|c|}{$10865 \pm 8$}} & 10857 & 10872 \\
\hline $6{ }^{1} \mathrm{~S}_{0}$ & & & & 11060 & 11070 \\
\hline $6^{3} \mathrm{~S}_{1}$ & $\Upsilon(11020)$ & \multicolumn{2}{|c|}{$11019 \pm 8$} & 11079 & 11089 \\
\hline $1{ }^{1} \mathrm{P}_{1}$ & & & & 9908 & 9918 \\
\hline $1^{3} \mathrm{P}_{2}$ & $\chi_{b 2}(1 P)$ & $9912.6 \pm 0.5)$ & \multirow{4}{*}{9900} & & \\
\hline $1^{3} \mathrm{P}_{1}$ & $\chi_{b 1}(1 P)$ & $9892.7 \pm 0.6$ & & 9908 & 9920 \\
\hline $1^{3} \mathrm{P}_{0}$ & $\chi_{b 0}(1 P)$ & $9859.9 \pm 1.0$ & & & \\
\hline $2{ }^{1} \mathrm{P}_{1}$ & & & & 10260 & 10279 \\
\hline $2{ }^{3} \mathrm{P}_{2}$ & $\chi_{b 2}(2 P)$ & $10268.5 \pm 0.4$ & \multirow{3}{*}{10260} & & \\
\hline $2{ }^{3} \mathrm{P}_{1}$ & $\chi_{b 1}(2 P)$ & $10255.2 \pm 0.5\}$ & & 10260 & 10280 \\
\hline $2{ }^{3} \mathrm{P}_{0}$ & $\chi_{b 0}(2 P)$ & $10232.1 \pm 0.6$ & & & \\
\hline
\end{tabular}

Shirkov-Solovtsov coupling constant, together with the running $u, d$ mass of eq. (27), the main difficulties seem to be solved. Even the masses of $\pi, \eta_{s}$ and $K$ turn correct without destroying the agreement in the other part of the spectrum. An exception could be represented by the masses of the $u \bar{b}$ channel that for some reason turn out to be high. The use of an effective running mass even for $m_{s}$ does not seem to produce any substantial modification. With reference to the mass of $\eta_{s}$ reported as a data in fig. 2 concerning the $s \bar{s}$ sector, it should be mentioned that the quantity is derived from the experimental values of $\eta$ and $\eta^{\prime}$ masses with conventional assumptions on the origin of this particles as a mixing of $\frac{1}{\sqrt{2}}(u \bar{u}+d \bar{d})$ and 
Table V. $c \bar{c}, n_{f}=4, \Lambda=0.2 \mathrm{GeV}$. (a) truncation prescription $\bar{\alpha}_{\mathrm{s}}=0.35$, $\sigma=0.2 \mathrm{GeV}^{2}, m_{c}=1.394 \mathrm{GeV}$. (b) Shirkov-Solovtsov $\alpha_{\mathrm{s}}\left(Q^{2}\right)$, $\sigma=0.18 \mathrm{GeV}^{2}, m_{c}=1.545 \mathrm{GeV}$

\begin{tabular}{|c|c|c|c|c|}
\hline States & & $\begin{array}{l}\text { experimental values } \\
(\mathrm{MeV})\end{array}$ & $\begin{array}{c}\text { (a) } \\
(\mathrm{MeV})\end{array}$ & $\begin{array}{c}\text { (b) } \\
(\mathrm{MeV}) \\
\end{array}$ \\
\hline $1{ }^{1} \mathrm{~S}_{0}$ & $\eta_{c}(1 S)$ & $2979.7 \pm 1.5$ & 2982 & 2977 \\
\hline $1{ }^{3} \mathrm{~S}_{1}$ & $J / \psi(1 S)$ & $3096.87 \pm 0.04$ & 3097 & 3097 \\
\hline $1 \Delta \mathrm{SS}$ & & 117 & 115 & 119 \\
\hline $2{ }^{1} \mathrm{~S}_{0}$ & $\eta_{c}(2 S)$ & $3594 \pm 5$ & 3575 & 3606 \\
\hline $2{ }^{3} \mathrm{~S}_{1}$ & $\psi(2 S)$ & $3685.96 \pm 0.09$ & 3642 & 3670 \\
\hline $2 \Delta \mathrm{SS}$ & & 92 & 67 & 64 \\
\hline $3{ }^{1} \mathrm{~S}_{0}$ & & & 3974 & 4005 \\
\hline $3{ }^{3} \mathrm{~S}_{1}$ & $\psi(4040)$ & $4040 \pm 10$ & 4025 & 4054 \\
\hline $4{ }^{1} \mathrm{~S}_{0}$ & & & 4298 & 4323 \\
\hline $4^{3} \mathrm{~S}_{1}$ & $\psi(4415)$ & $4415 \pm 6$ & 4341 & 4364 \\
\hline $1{ }^{1} \mathrm{P}_{1}$ & & & 3529 & 3556 \\
\hline $1^{3} \mathrm{P}_{2}$ & $\chi_{c 2}(1 P)$ & $3556.18 \pm 0.13)$ & & \\
\hline $1^{3} \mathrm{P}_{1}$ & $\chi_{c 1}(1 P)$ & $3510.51 \pm 0.12\} 3525$ & 3530 & 3561 \\
\hline $1^{3} \mathrm{P}_{0}$ & $\chi_{c 0}(1 P)$ & $3415.1 \pm 0.8$ & & \\
\hline $2{ }^{1} \mathrm{P}_{1}$ & & & 3925 & 3954 \\
\hline $2^{3} \mathrm{P}$ & & & 3927 & 3958 \\
\hline $1{ }^{1} \mathrm{D}_{2}$ & & & 3813 & 3853 \\
\hline $1{ }^{3} \mathrm{D}_{3}$ & & & & \\
\hline $1^{3} \mathrm{D}_{2}$ & $\psi(3836)$ & $3836 \pm 13$ & 3813 & 3854 \\
\hline $1^{3} \mathrm{D}_{1}$ & $\psi(3770)$ & $3769.9 \pm 2.5)$ & & \\
\hline $2{ }^{1} \mathrm{D}_{2}$ & & & 4149 & 4183 \\
\hline $2^{3} \mathrm{D}_{3}$ & & & & \\
\hline $2{ }^{3} \mathrm{D}_{2}$ & & & 4149 & 4184 \\
\hline $2^{3} \mathrm{D}_{1}$ & $\psi(4160)$ & $4159 \pm 20$ & & \\
\hline
\end{tabular}

$s \bar{s}$ states.

\section{Conclusions}

In conclusion, starting from a reasonable ansatz on the Wilson loop it is possible to develop a second order Bethe-Salpeter approach to the $q \bar{q}$ bound state problem. From this, by a standard reduction technique, an eigenvalue equation for a mass squared operator $M^{2}=\left(w_{1}+w_{2}\right)^{2}+U$ is obtained, that can be effectively applied to the calculation of the spectrum. 
Table VI. Light-heavy quarkonium systems, $n_{f}=4, \Lambda=0.2 \mathrm{GeV}$. (a) truncation prescription $\bar{\alpha}_{\mathrm{s}}=0.35, \sigma=0.2 \mathrm{GeV}^{2}, m_{b}=4.763 \mathrm{GeV}$, $m_{c}=1.394 \mathrm{GeV}, m_{u}=m_{d}=0.01 \mathrm{GeV}$. (b) Shirkov-Solovtsov $\alpha_{\mathrm{s}}\left(Q^{2}\right)$, $\sigma=0.18 \mathrm{GeV}^{2}, m_{b}=4.898 \mathrm{GeV}, m_{c}=1.545 \mathrm{GeV}$, $m_{u}^{2}=m_{d}^{2}=0.121 k-0.025 k^{2}+0.25 k^{4}$.

\begin{tabular}{|c|c|c|c|c|}
\hline States & & $\begin{array}{c}\text { experimental values } \\
(\mathrm{MeV})\end{array}$ & $\begin{array}{c}(\mathrm{a}) \\
(\mathrm{MeV})\end{array}$ & $\begin{array}{c}(\mathrm{b}) \\
(\mathrm{MeV})\end{array}$ \\
\hline \multicolumn{5}{|c|}{$\overline{q \bar{c}(q=u, d)}$} \\
\hline $1{ }^{1} \mathrm{~S}_{0}$ & $\left\{\begin{array}{l}D^{ \pm} \\
D^{0}\end{array}\right.$ & $\left.\begin{array}{l}1869.3 \pm 0.5 \\
1864.5 \pm 0.5\end{array}\right\}$ & 1875 & 1862 \\
\hline $1{ }^{3} \mathrm{~S}_{1}$ & $\left\{\begin{array}{l}D^{*}(2010)^{ \pm} \\
D^{*}(2007)^{0}\end{array}\right.$ & $\left.\begin{array}{l}2010.0 \pm 0.5 \\
2006.7 \pm 0.5\end{array}\right\}$ & 2020 & 2012 \\
\hline $1 \Delta \mathrm{SS}$ & & $141 \pm 1$ & 145 & 150 \\
\hline $2{ }^{1} \mathrm{~S}_{0}$ & $D^{\prime}$ & 2580 & 2525 & 2615 \\
\hline $2^{3} \mathrm{~S}_{1}$ & $D^{* \prime}$ & $2637 \pm 8$ & 2606 & 2683 \\
\hline $2 \Delta \mathrm{SS}$ & & 57 & 81 & 67 \\
\hline $1^{1} \mathrm{P}_{1}$ & & & 2474 & 2504 \\
\hline $1^{3} \mathrm{P}_{2}$ & $\left\{\begin{array}{l}D_{2}^{*}(2460)^{ \pm} \\
D_{2}^{*}(2460)^{0}\end{array}\right.$ & $\left.\begin{array}{c}2459 \pm 4 \\
2458.9 \pm 2.0\end{array}\right)$ & \multirow{3}{*}{2475} & \multirow{3}{*}{2511} \\
\hline $1^{3} \mathrm{P}_{1}$ & $\left\{\begin{array}{c}D_{1}(2420)^{ \pm} \\
D_{1}(2420)^{0}\end{array}\right.$ & $\begin{aligned} 2427 & \pm 5 \\
2422.2 & \pm 1.8\end{aligned}$ & & \\
\hline $1^{3} \mathrm{P}_{0}$ & & & & \\
\hline \multicolumn{5}{|c|}{$q \bar{b}(q=u, d)$} \\
\hline $1{ }^{1} \mathrm{~S}_{0}$ & $\left\{\begin{array}{l}B^{ \pm} \\
B^{0}\end{array}\right.$ & $\left.\begin{array}{l}5278.9 \pm 1.8 \\
5279.2 \pm 1.8\end{array}\right\}$ & 5273 & 5315 \\
\hline $1{ }^{3} \mathrm{~S}_{1}$ & $B^{*}$ & $5324.8 \pm 1.8$ & 5339 & 5376 \\
\hline $1 \Delta \mathrm{SS}$ & & $46 \pm 3$ & 66 & 61 \\
\hline $2{ }^{1} \mathrm{~S}_{0}$ & & & 5893 & 6034 \\
\hline $2^{3} \mathrm{~S}_{1}$ & $B^{* \prime}$ & $5906 \pm 14$ & 5933 & 6064 \\
\hline $1^{1} \mathrm{P}_{1}$ & & & 5791 & 5838 \\
\hline $1^{3} \mathrm{P}$ & & $5825 \pm 14$ & 5792 & 5841 \\
\hline
\end{tabular}

If only the one gluon exchange contribution is included in the perturbative part of the Bethe-Salpeter kernel, but a truncated running constant $\alpha_{\mathrm{s}}\left(Q^{2}\right)$ is used, a reasonable good reproduction of the data is already obtained (as far as calculable) with the relevant exception of the light pseudo scalar mesons. However if the Shirkov-Solovtsov prescription for $\alpha_{\mathrm{s}}\left(Q^{2}\right)$ 
Table VII. Light-heavy quarkonium systems, $n_{f}=4, \Lambda=0.2 \mathrm{GeV}$. (a) truncation prescription $\bar{\alpha}_{\mathrm{s}}=0.35, \sigma=0.2 \mathrm{GeV}^{2}, m_{b}=4.763 \mathrm{GeV}$, $m_{c}=1.394 \mathrm{GeV}, m_{s}=0.2 \mathrm{GeV}$. (b) Shirkov-Solovtsov $\alpha_{\mathrm{s}}\left(Q^{2}\right)$, $\sigma=0.18 \mathrm{GeV}^{2}, m_{b}=4.898 \mathrm{GeV}, m_{c}=1.545 \mathrm{GeV}, m_{s}=0.381 \mathrm{GeV}$.

\begin{tabular}{ccccc}
\hline States & & $\begin{array}{c}\text { experimental values } \\
(\mathrm{MeV})\end{array}$ & $\begin{array}{c}(\mathrm{a}) \\
(\mathrm{MeV})\end{array}$ & $\begin{array}{c}(\mathrm{b}) \\
(\mathrm{MeV})\end{array}$ \\
\hline$s \bar{c}$ & & & \\
$1{ }^{1} \mathrm{~S}_{0}$ & $D_{s}^{ \pm}$ & $1968.5 \pm 0.6$ & 1982 & 1953 \\
$1{ }^{3} \mathrm{~S}_{1}$ & $D_{s}^{* \pm}$ & $2112.4 \pm 0.7$ & 2120 & 2102 \\
$1 \Delta \mathrm{SS}$ & & $144 \pm 1$ & 138 & 149 \\
$2{ }^{1} \mathrm{~S}_{0}$ & & & 2617 & 2610 \\
$2{ }^{3} \mathrm{~S}_{1}$ & & & 2698 & 2691 \\
\hline $1{ }^{1} \mathrm{P}_{1}$ & & 2547 & 2555 \\
$1{ }^{3} \mathrm{P}_{2}$ & $D_{s J}(2573)^{ \pm}$ & $2573.5 \pm 1.7$ & & \\
$1{ }^{3} \mathrm{P}_{1}$ & $D_{s 1}(2536)^{ \pm}$ & $2535.35 \pm 0.34$ & 2548 & 2562 \\
$1{ }^{3} \mathrm{P}_{0}$ & & & & \\
\hline$s \bar{b}$ & & & & \\
$1{ }^{1} \mathrm{~S}_{0}$ & $B_{s}^{0}$ & $5369.3 \pm 2.0$ & 5364 & 5348 \\
$1{ }^{3} \mathrm{~S}_{1}$ & $B_{s}^{*}$ & $5416.3 \pm 3.3$ & 5429 & 5416 \\
$1 \Delta \mathrm{SS}$ & & $47 \pm 4$ & 65 & 68 \\
$2{ }^{1} \mathrm{~S}_{0}$ & & & 5985 & 5969 \\
$2{ }^{3} \mathrm{~S}_{1}$ & & & 6024 & 6009 \\
\hline $1{ }^{1} \mathrm{P}_{1}$ & & 5858 & 5857 \\
$1{ }^{3} \mathrm{P}$ & $B_{s J}^{*}(5850)$ & $5853 \pm 15$ & 5859 & 5860 \\
\hline
\end{tabular}

is adopted and a phenomenological running mass is used for the $u$ and $d$ quarks, even the light pseudo scalar mesons occur in the correct range.

An open problem is a resolution of the complicated Dyson-Schwinger equation in some reasonable approximation (19) and an actual derivation of an equation of the type (27) from first principle.

\section{References}

1. N. Brambilla, E. Montaldi, G.M. Prosperi, Phys. Rev. D 54 (1996) 3506; G.M. Prosperi, in Problems of Quantum Theory of Fields, Pag. 381, B.M. Barbashov, G.V. Efimov, A.V. Efremov Eds. JINR Dubna 1999, hepph/9906237.

2. For a different approach: Yu.S. Kalashnikova, A.V. Nefediev, Yu.A. Simonov, Phys. Rev. D 64 (2001) 014037; Yu.S. Kalashnikova, A.V. Nefediev, Phys. Lett. B 530 (2002) 117, and references therein. 
3. M. Baldicchi, G.M. Prosperi, Phys. Rev. D 62 (2000) 114024; Fizika B 8 (1999) 2, 251; M. Baldicchi, in "QCD: Perturbative or Nonperturbative ?" Pag. 325, L. S. Ferreira, P. Nogueira, J. I.Silva-Marcos Eds. World Scientific (2001), hep-ph/9911268.

4. A.I. Sanda Phys. Rev. Lett. 42 (1979) 1658; Yu.L. Dokshitzer, A. Lucenti, G. Marchesini, G.P. Salam, JHEP 9805 (1998) 003; Yu.L. Dokshitzer, in $29^{\text {th }}$ International conference on High-Energy Physics (ICHE 98), Vancouver, Canada, A. Astbury, D. Axen, J Robinson Eds. World Scientific (1999), hepph/9812252; Yu.L. Dokshitzer, V.A. Khoze, S.I. Troyan, Phys. Rev. D 53 (1996) 89.

5. D.V. Shirkov, I.L. Solovtsov, Phys. Rev. Lett. 79 (1997) 1209; Theor. Math. Phys. 120 (1999) 1220; K.A. Milton, I.L. Solovtsov, O.P. Solovtsova, Phys. Rev. D 64 (2001) 016005; N.G.Stefanis, W. Schroers, H.-Ch. Kim, Phys. Lett. B 449 (1999) 299, see also Eur. Phys. J. C 18 (2000) 137; A. V. Nesterenko, Phys. Rev. D 62 (2000) 094028; D 64 (2001) 116009; A. V. Nesterenko, Mod. Phys. Lett. A 15 (2000) 2401; A. V. Nesterenko, I.L. Solovtsov, ibid. A 16 (2001) 2517; S. J. Brodsky, S. Menke, C. Merino, Phys. Rev. D 67 (2003), 055008.

6. M. Baldicchi and G. M. Prosperi Phys. Rev. D 66 (2002) 074008.

7. M.B. Hecht, C.D. Roberts, S.M. Schmidt, Phys. Rev. C 63 (2001) 025213, and references therein.

8. A. Barchielli, E. Montaldi, G.M. Prosperi, Nucl. Phys. B 296 (1988) 625; Erratum-ibid. B 303 (1988) 752; A. Barchielli, N. Brambilla, G.M. Prosperi, Il Nuovo Cimento 103 A (1990) 59; N. Brambilla, P. Consoli, G.M. Prosperi, Phys. Rev. D 50 (1994) 5878.

9. The Review of Particle Physics, K. Hagiwara et al., Phys. Rev. D 66010001 (2002). 